\title{
THE EFFECTS OF THE INSERTION DEVICES AT THE SUPER SOR LIGHT SOURCE
}

\author{
Kentaro Harada ${ }^{\dagger}$, Masanori Satoh, Hiroyuki Takaki, Tadashi Koseki, Norio Nakamura, \\ and Yukihide Kamiya ${ }^{* 1}$ \\ ISSP SRL, 5-1-5 Kashiwanoha, Kashiwa, Chiba, 277-8581, Japan \\ ${ }^{* 1}$ KEK, 1-1 Oho Tsukuba, Ibaraki 305-0801, Japan
}

\section{Abstract}

The Super SOR ring is a third generation light source constructed at Kashiwa Campus of the University of Tokyo. In this paper, we present the formalization of the circularly polarizing undulator and the scheme of beam optics correction.

\section{OVERVIEW OF THE RING}

The Super SOR ring [1] is a third-generation VUV and soft X-ray light source which has two modes of optics: the ultra-low emittance mode $(1.0 \mathrm{GeV}, \quad 0.7$ nm•rad emittance), and the low emittance mode $(1.0 \mathrm{GeV}, 2.2$ nm•rad; $1.6 \mathrm{GeV}, 5.6 \mathrm{~nm} \bullet \mathrm{rad})$. The ring has about $250 \mathrm{~m}$ circumference and twelve straight sections, two of which are $29 \mathrm{~m}$ long. One of the $29 \mathrm{~m}$ long straight sections is configured as saw-tooth to use four undulators simultaneously. The main character of the ring is the realization of subnanometer emittance and the insertion of $27 \mathrm{~m}$ very long undulator. That is, for ultra low emittance mode, its emittance is below the diffraction limit for the

Table 1: Main parameters of the ring

\begin{tabular}{|c|c|c|c|c|}
\hline & & $\begin{array}{l}\text { U ltra Low } \\
\text { em ittance }\end{array}$ & \multicolumn{2}{|c|}{ Low Em ittance } \\
\hline Energy & $\mathrm{GeV}$ & 1 & 1 & 1.6 \\
\hline C ircum ference & $\mathrm{m}$ & 249.38 & 249.38 & 249.38 \\
\hline Em ittance & $\mathrm{nm} \mathrm{rad}$ & 0.75 & 2.20 & 5.65 \\
\hline Energy Spread & & $4.91 \mathrm{E}-04$ & $4.91 \mathrm{E}-04$ & $7.85 \mathrm{E}-04$ \\
\hline $\begin{array}{l}\text { M om entum Com paction } \\
\text { Factor }\end{array}$ & & $5.69 \mathrm{E}-04$ & $11.0 \mathrm{E}-04$ & $11.0 \mathrm{E}-04$ \\
\hline \multicolumn{5}{|l|}{ Betatron Tune } \\
\hline horizonta1 & & 19.40 & 14.26 & 14.26 \\
\hline vertical & 3 & 8.71 & 12.19 & 12.19 \\
\hline \multicolumn{5}{|l|}{ Chrom a tic ity } \\
\hline horizonta1 & $\mathrm{n}$ & -37.1 & -39.5 & -39.5 \\
\hline vertical & 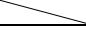 & -39.5 & -21.7 & -21.7 \\
\hline M agnetic F ield of Bend. & $\mathrm{T}$ & 1.092 & 1.092 & 1.747 \\
\hline Curvature Radius of Bend. & $\mathrm{m}$ & 3.056 & 3.056 & 3.056 \\
\hline $\begin{array}{l}\text { Critica1Photon Energy of } \\
\text { Bend. }\end{array}$ & $\mathrm{eV}$ & 726 & 726 & 2973 \\
\hline Energy Loss / tum & $\mathrm{keV}$ & 28.9 & 28.9 & 189.7 \\
\hline \multicolumn{5}{|l|}{ Radiation Dam ping $\mathrm{T}$ im $\mathrm{e}$} \\
\hline horizonta1 & $\mathrm{m} \mathrm{sec}$ & 57.2 & 572 & 14 \\
\hline vertical & $\mathrm{m} \mathrm{sec}$ & 57.5 & 57.5 & 14 \\
\hline longitud ina1 & $\mathrm{m} \mathrm{sec}$ & 28.8 & 28.8 & 7 \\
\hline Revolution Frequency & $\mathrm{MHz}$ & 1.202 & 1202 & 1202 \\
\hline RF Frequency & $\mathrm{MHz}$ & 500.1 & 500.1 & 500.1 \\
\hline Harm on ic Num ber & 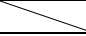 & 416 & 416 & 416 \\
\hline RF Voltage & M V & 0.7 & 0.7 & 1.4 \\
\hline Synchrotron Tune & $\mathrm{N}$ & 0.0051 & 0.0072 & 0.0079 \\
\hline Bunch Length & $\mathrm{mm}$ & 2.16 & 2.99 & 4.3 \\
\hline RF Bucker H ight & & 0.042 & 0.0302 & 0.0312 \\
\hline
\end{tabular}

$\overline{{ }^{\dagger} k e n t a r o @ i s s p . u-t o k y o . a c . j p}$ photon of about $100 \mathrm{eV}$ and the brilliance of the light from $27 \mathrm{~m}$ undulator is over $10^{20}$ photons $/ \mathrm{s} / \mathrm{mm}^{2} / \mathrm{mrad}^{2} / 0.1 \%$ b.w..

We adopted 24 normal cells of the theoretical minimum type for the lattice design of the Super SOR ring, and then designed the optics of the straight sections so that each straight section inserted between normal cells looks transparent for the non-linear effects of sextupoles. To keep large dynamic aperture with the insertion devices and the magnetic errors, we make correction to maintain 24-fold symmetry of normal cells and the transparency of long straight sections for on-momentum particles [2]. Indeed for insertion devices, we correct the optics to keep the tune advance of the straight section constant by using the quadrupole magnets at the matching section. [4]

The plan view of the ring is shown in Fig. 1 and the main parameters of the ring are given in Table 1.

\section{FORMALIZATION OF CIRCULARLY POLARIZING UNDULATOR}

The magnetic fields in the undulators are

$$
\begin{aligned}
& B_{x}=B_{0 y}\left(\frac{k_{y}^{\prime}}{k_{y}}\right) \sinh k_{y}^{\prime} x \sinh k_{y} y \cos k z \\
& \quad+B_{0 x} \cosh k_{x} x \cosh k_{x}^{\prime} y \sin k z \\
& B_{y}=B_{0 y} \cosh k_{y}^{\prime} x \cosh k_{y} y \cos k z \\
& \quad+B_{0 x}\left(\frac{k_{x}^{\prime}}{k_{x}}\right) \sinh k_{x} x \sinh k_{x}^{\prime} y \sin k z \\
& B_{z}=B_{0 y}\left(\frac{k}{k_{y}}\right) \cosh k_{y}^{\prime} x \sinh k_{y} y \sin k z \\
& \quad+B_{0 x}\left(\frac{k}{k_{x}}\right) \sinh k_{x} x \cosh k_{x}^{\prime} y \cos k z
\end{aligned}
$$

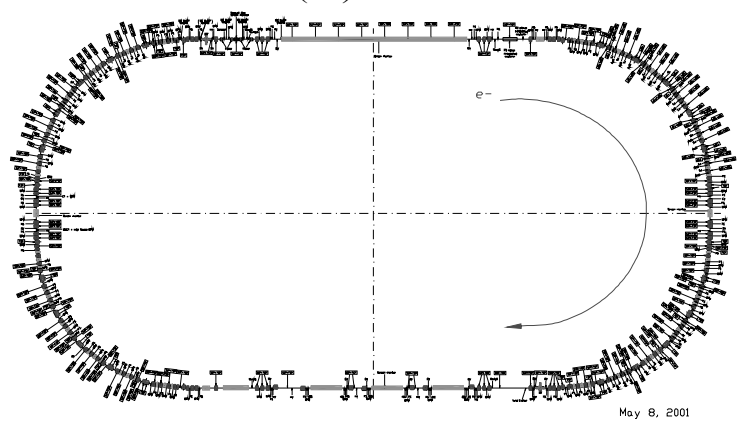

Figure 1: Plan view of the ring 
$\mathrm{B}_{0 \mathrm{x}}$ and $\mathrm{B}_{0 \mathrm{y}}$ are the peak magnetic fields of the horizontal and vertical directions, $k=2 \pi / \lambda_{u}, \lambda_{u}$ is the period of the undulator, and $\mathrm{k}_{\mathrm{x}}, \mathrm{k}_{\mathrm{x}}^{\prime}, \mathrm{k}_{\mathrm{y}}, \mathrm{k}_{\mathrm{y}}$ are the transverse wave numbers which satisfy $\mathrm{k}_{\mathrm{x}}{ }^{2}+\mathrm{k}_{\mathrm{x}}{ }^{2}=\mathrm{k}^{2}$ and $\mathrm{k}_{\mathrm{y}}{ }^{2}+\mathrm{k}_{\mathrm{y}}{ }^{2}=\mathrm{k}^{2}$ from Maxwell equation. Substituting these field equations for the equations of motions of the particle, dividing the motion of particle into rapid part (central orbit) and slow part (betatron oscillation), averaging along longitudinal direction, we finally get the equations of slow part of oscillations [5] as

$$
\begin{aligned}
& x^{\prime \prime}=-\frac{k_{y}^{\prime}}{4 \rho_{y}{ }^{2} k^{2}} \sinh 2 k_{y}^{\prime} x+\frac{k_{x}{ }^{2}}{4 \rho_{x}{ }^{2} k^{2} k_{x}} \sinh 2 k_{x} x \\
& -\frac{k_{y}^{\prime}}{4 \rho_{y}{ }^{2} k_{y}^{2}} \sinh 2 k_{y}^{\prime} x \sinh ^{2} k_{y} y-\frac{1}{4 \rho_{x}^{2} k_{x}} \sinh 2 k_{x} x \cosh ^{2} k_{x}{ }^{y} \\
& -\frac{y^{\prime}}{\rho_{x} \rho_{y} k} \frac{k_{x}^{\prime} k_{y}^{\prime}}{k_{x} k_{y}} \sinh k_{y}^{\prime} x \sinh k_{y} y \sinh k_{x} x \sinh k_{y}^{\prime} y \\
& +\frac{y^{\prime}}{\rho_{x} \rho_{y} k} \cosh k_{y}^{\prime} x \cosh k_{y} y \cosh k_{x} x \cosh k_{y}^{\prime} y \\
& y^{\prime \prime}=-\frac{k_{x}^{\prime}}{4 \rho_{x}^{2} k^{2}} \sinh 2 k_{x}^{\prime} y+\frac{k_{y}{ }^{2}}{4 \rho_{y}{ }^{2} k^{2} k_{y}} \sinh 2 k_{y} y \\
& -\frac{1}{4 \rho_{y}{ }^{2} k_{y}} \cosh ^{2} k_{y}^{\prime} x \sinh 2 k_{y} y-\frac{k_{x}^{\prime}}{4 \rho_{y}{ }^{2} k_{x}{ }^{2}} \sinh ^{2} k_{x} x \sinh 2 k_{x}{ }_{x} y \\
& +\frac{x^{\prime}}{\rho_{x} \rho_{y} k} \frac{k_{x}^{\prime} k_{y}^{\prime}}{k_{x} k_{y}} \sinh k_{y}^{\prime} x \sinh k_{y} y \sinh k_{x} x \sinh k_{y}^{\prime} y \\
& -\frac{x}{\rho_{x} \rho_{y} k} \cosh k_{y}^{\prime} x \cosh k_{y} y \cosh k_{x} x \cosh k_{y}^{\prime} y \text {. }
\end{aligned}
$$

Here, $\rho_{\mathrm{x}}$ and $\rho_{\mathrm{y}}$ are the curvature radius of peak magnetic fields. If we take the linear part of these equations, the equations of betatron oscillations are

$$
\begin{aligned}
& x "=-\left(\frac{k_{y}{ }^{2}}{2 \rho_{y}{ }^{2} k^{2}}+\frac{k_{x}{ }^{2}}{2 \rho_{x}{ }^{2} k^{2}}\right) x+\frac{y^{\prime}}{\rho_{x} \rho_{y} k} \\
& y^{\prime \prime}=-\left(\frac{k_{x}{ }^{2}}{2 \rho_{x}{ }^{2} k^{2}}+\frac{k_{y}{ }^{2}}{2 \rho_{y}{ }^{2} k^{2}}\right) y-\frac{y^{\prime}}{\rho_{x} \rho_{y} k} .
\end{aligned}
$$

If the fields are upper concave in the horizontal or vertical direction, they act the focusing force on the beam. If the fields are lower concave, substituting imaginary wave number into all the equations mentioned above, they give the defocusing force.

By using Laplace transform, the transfer matrix of linear equation above are as follows.

$$
\begin{aligned}
M(1,1)= & \frac{\alpha+\beta-\left(a_{y}+b^{2}\right)}{2 \beta} \cos (\sqrt{\alpha+\beta} L) \\
& -\frac{\alpha-\beta-\left(a_{y}+b^{2}\right)}{2 \beta} \cos (\sqrt{\alpha-\beta} L) \\
M(1,2)= & \frac{\alpha+\beta-a_{y}}{2 \beta \sqrt{\alpha+\beta}} \sin (\sqrt{\alpha+\beta} L)-\frac{\alpha-\beta-a_{y}}{2 \beta \sqrt{\alpha-\beta}} \sin (\sqrt{\alpha-\beta} L) \\
M(1,3)= & \frac{b a_{y}}{2 \beta \sqrt{\alpha+\beta}} \sin (\sqrt{\alpha+\beta} L)-\frac{b a_{y}}{2 \beta \sqrt{\alpha-\beta}} \sin (\sqrt{\alpha-\beta} L)
\end{aligned}
$$

$$
M(1,4)=\frac{-b}{2 \beta} \cos (\sqrt{\alpha+\beta} L)+\frac{b}{2 \beta} \cos (\sqrt{\alpha-\beta} L)
$$

Here,

$$
\begin{aligned}
& a_{x}=\frac{k_{y}{ }^{2}}{2 \rho_{y}{ }^{2} k^{2}}+\frac{k_{x}{ }^{2}}{2 \rho_{x}{ }^{2} k^{2}}, a_{y}=\frac{k_{x}{ }^{2}}{2 \rho_{x}{ }^{2} k^{2}}+\frac{k_{y}{ }^{2}}{2 \rho_{y}{ }^{2} k^{2}}, \\
& b=\frac{y^{\prime}}{\rho_{x} \rho_{y} k}, \alpha=\frac{1}{2}\left(a_{x}+a_{y}+b^{2}\right), \beta=\sqrt{\alpha^{2}-a_{x} a_{y}} .
\end{aligned}
$$

To derive the expression of $\mathrm{M}(2, *)$, we differentiate $\mathrm{M}\left(1,{ }^{*}\right)$ with respect to $\mathrm{L}$, and to derive $\mathrm{M}\left(3,{ }^{*}\right)$ and $\mathrm{M}\left(4,{ }^{*}\right)$, we exchange $\mathrm{x}$ with $\mathrm{y}$ and $\mathrm{b}$ with $-\mathrm{b}$.

\section{THE EFFECTS OF UNDULATORS}

\section{1 horizontally polarizing undulators}

For horizontally polarizing undulators $\left(\mathrm{B}_{0 \mathrm{x}}=0\right)$, from the field calculation result, the magnetic fields of the undulators have almost no wave number dependence in the horizontal direction and thus $\mathrm{k}_{\mathrm{y}}^{\prime}=0$ and $\mathrm{B}_{\mathrm{x}}=0$ (i.e. $\mathrm{k}_{\mathrm{y}}=\mathrm{k}$ ). No horizontal force exerted on the beam and the linear part (the vertical focusing force) only depends on the peak magnetic field $\mathrm{B}_{0 \mathrm{y}}$. This case is already treated in reference [3].
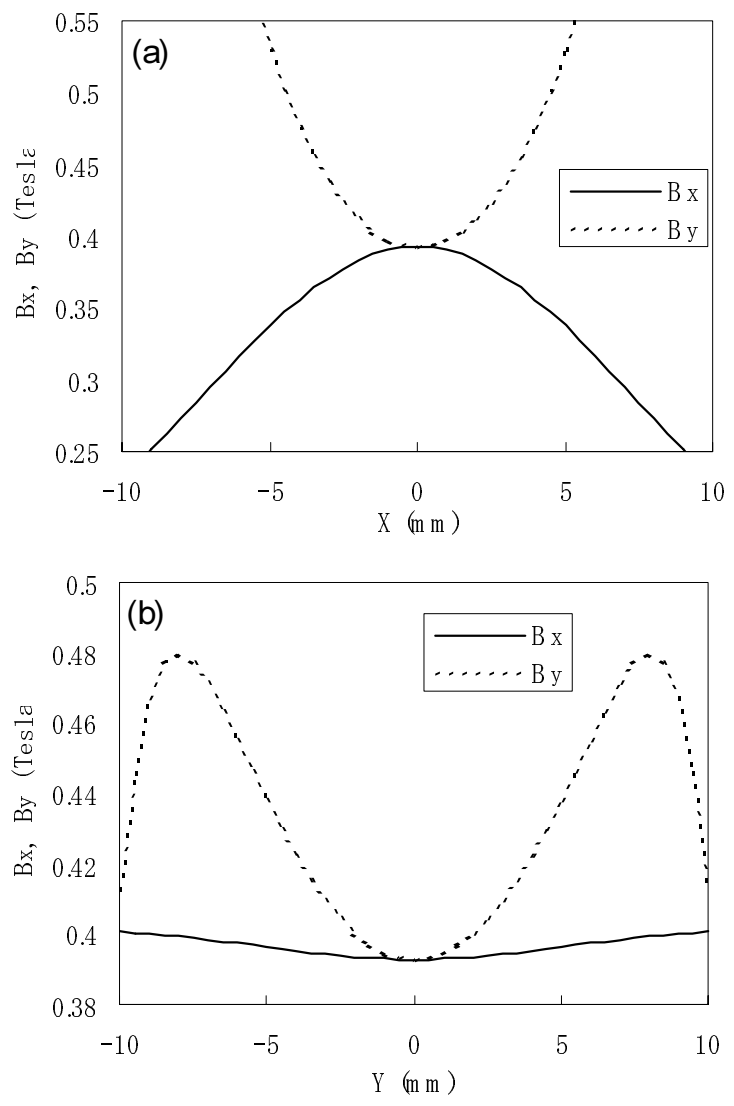

Figure 2: Magnetic field of U60N

$\lambda_{\mathrm{u}}=60 \mathrm{~mm}$ and $\mathrm{B}_{0 \mathrm{x}}=\mathrm{B}_{0 \mathrm{y}}=0.39 \mathrm{~T}$. (a) is the field at $\mathrm{z}=15 \mathrm{~mm}$ ( $\sin \mathrm{kz}$ part), and (b) at $\mathrm{z}=0 \mathrm{~mm}$ (cos kz part). 


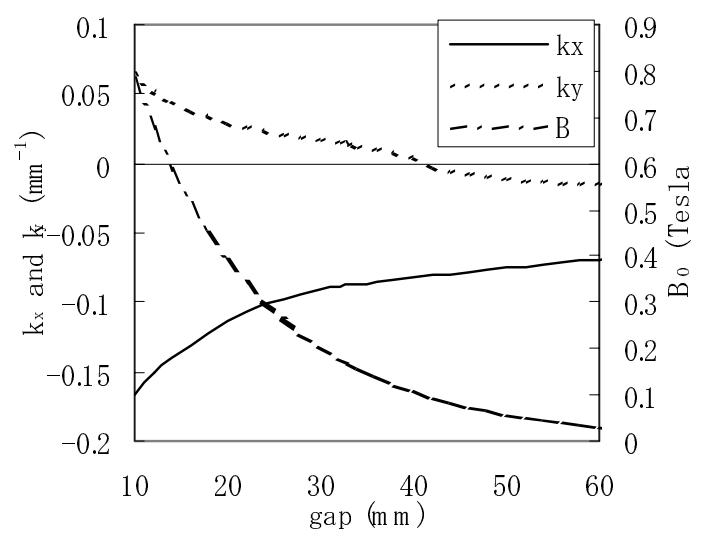

Figure 3: gap and wave number

Imaginary wave number is showed as negative wave number in convenience. $\mathrm{k}_{\mathrm{x}}$ is always imaginary and $\mathrm{k}_{\mathrm{y}}$ becomes imaginary when the gap is larger than $42 \mathrm{~mm}$. (Actually the minimum value of the gap is $20 \mathrm{~mm}$.)
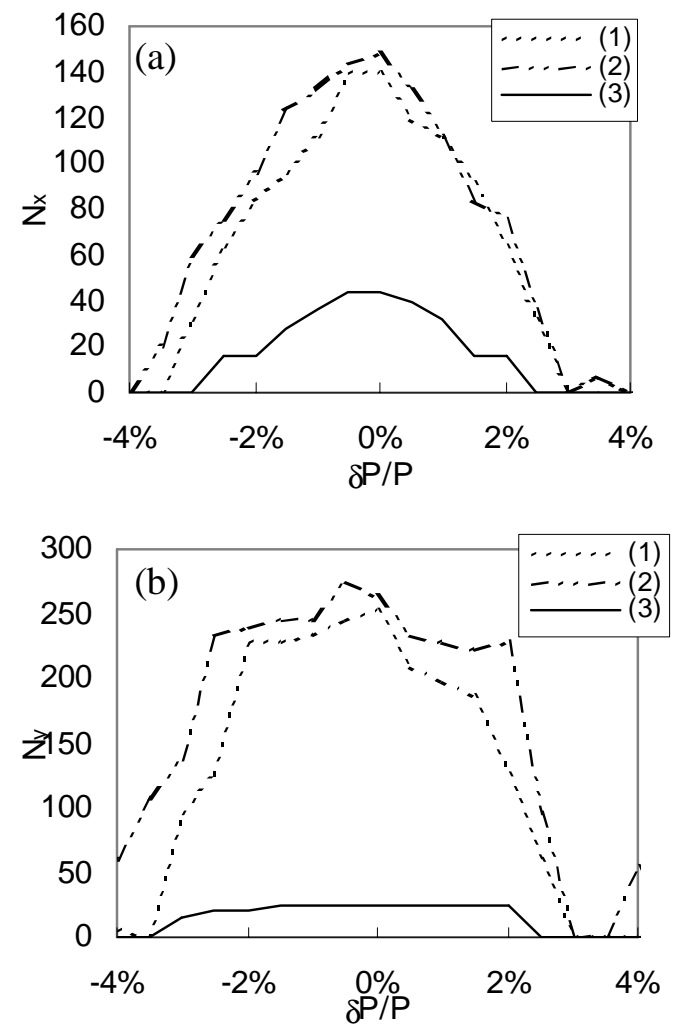

Figure 4 : dynamic aperture with U60N

(a) and (b) show the horizontal and vertical dynamic apertures in the $1.6 \mathrm{GeV}$ low emittance mode with U60N. $\mathrm{N}_{\mathrm{x}}$ and $\mathrm{N}_{\mathrm{y}}$ are the emittance normalized beam size. (1) is for horizontally polarizing case with linear effect, (2) circularly polarizing case with linear effect, and (3) circularly polarizing case with non-linear effect. In the case (3), the magnetic fields are assumed to be exponentially increasing, and thus the dynamic aperture (about $8 \mathrm{~mm}$ ) becomes almost the half width of the undulator gap $(10 \mathrm{~mm})$.

\section{2 circularly polarizing undulators}

For circularly polarizing undulators $\left(\mathrm{B}_{0 \mathrm{x}}=\mathrm{B}_{0 \mathrm{y}}\right)$, we adopted an Apple-2 type undulator [5] and from the field calculation, the magnetic fields have large wave number dependence in both horizontal and vertical directions. All of $\mathrm{k}_{\mathrm{x}}, \mathrm{k}_{\mathrm{x}}, \mathrm{k}_{\mathrm{y}}, \mathrm{k}_{\mathrm{y}}$ are finite (non-zero) values and the focusing force depends on them. In order to calculate this force precisely, transverse wave numbers should be obtained by comparing the formulae (1) with the field calculation results (like Fig.2). The relation between the undulator gap and wave numbers obtained are shown in Fig. 3.

If we consider only linear effect of insertion devices, whether the magnetic field is upper concave (imaginary wave number and defocusing force) or lower concave (real wave number and focusing force), the dynamic aperture after optics correction is not much changed from that without insertion devices. With non-linear effect, describing the magnetic field as exponential function, if the linear optics correction is done, the dynamic aperture is decreased but as large as the physical aperture (see Fig.4).

\section{CONCLUSION}

In order to keep large dynamic aperture at the Super SOR ring, the tune advance of straight sections must be kept constant against the effect of the insertion devices. So we should precisely estimate the linear effect (focusing or defocusing force on the beam) from the field calculation, because the magnetic field of the circularly polarizing undulators are much different from the ideal case and has large dependence on horizontal and vertical wave numbers.

\section{REFERENCES}

[1] http://www.issp.u-tokyo.ac.jp/labs/sor/index-e.html [2] K.Harada, M.Satoh, H.Takaki, T.Koseki, N.Nakamura, Y.Kamiya and Y.Kobayashi," The lattice of the VSX light source" , accepted for publication in Nucl. Instrum. Mathods A, (maybe vol. 467-468, 2001)

[3] K.Harada, M.Satoh, H.Takaki, T.Koseki, N.Nakamura and Y.Kamiya," The effects of the insertion devices at the VSX light source", accepted for publication in Nucl. Instrum. Mathods A, (maybe vol. 467-468, 2001)

[4] Y. Kamiya, Lecture notes of the insertion devices and the beam dynamics, (unpublished) and H. Tanaka, ibid.

[5] N.Nakamura et al. "Three-Dimensional Magnetic Field Analysis of a Variably Polarizing Undulator", in these proceedings 